\title{
Biodiversity of Holocene marine fish of the southeast coast of Brazil
}

\author{
Augusto Barros Mendes $^{I}$, Michelle Rezende Duarte ${ }^{I^{*}}$ \& Edson Pereira Silva ${ }^{I}$ \\ ${ }^{1}$ Universidade Federal Fluminense, Departamento de Biologia Marinha, Niterói, Brazil. \\ *Corresponding author: Michelle Rezende Duarte, e-mail: michellerezendeduarte@yahoo.com.br
}

\begin{abstract}
MENDES, A. B., DUARTE, M. R., SILVA, E. P. Biodiversity of Holocene marine fish of the southeast coast of Brazil. Biota Neotropica. 18(1): e20170394. http://dx.doi.org/10.1590/1676-0611-BN-2017-0394
\end{abstract}

\begin{abstract}
Middens are archaeological sites dating between 8,000 and 1,000 years before present and are commonly found on the Brazilian coast. Data were collected from 68 middens allowing an inventory of 142 fish species, most of them recorded in no more than five sites. Conversely, Micropogonias furnieri and Pogonias cromis had the highest frequencies of occurrence. The biogeographic, ecological and economic data showed that most of the identified fish are widely distributed in the Western Atlantic (59.72\%) and inhabit estuarine environments (53.99\%), while most species have a demersal habit (35.92\%) and exhibit oceanic migratory behaviour (28.87\%). Lastly, the surveyed fish are predominantly carnivorous $(72.54 \%)$ with some commercial value $(96.48 \%)$. Chi-squared tests comparing midden inventory and current ichthyofauna checklists failed to show significant differences between them $(p>0.99)$. Thus, the results indicate that zoo-archaeological fish remains are key evidence of Holocene biodiversity and may help the establishment of more complete baselines.
\end{abstract}

Keywords: Baselines, Fishermen-Gatherers-Hunters, Ichthyofauna, Middens, Species richness, Zooarchaeology.

\section{Biodiversidade de peixes marinhos do Holoceno da costa Sudeste brasileira}

\begin{abstract}
Resumo: Sambaquis são sítios arqueológicos, datando entre 8,000 e 1,000 anos antes do presente, encontrados na costa brasileira. Neste trabalho, dados ictiológicos referentes a 68 sambaquis da costa Sudeste do Brasil foram compilados e construído um inventário de referência no qual constam 142 espécies, a maioria das quais com registro em não mais que cinco sítios. Por outro lado, as espécies Micropogonias furnieri e Pogonias cromis apresentaram alta frequência de ocorrência nos sambaquis. Os dados biogeográficos, ecológicos e econômicos mostraram que a maioria das espécies registradas nos sambaquis são de ampla distribuição no Atlântico Ocidental (59.72\%), habitam ambientes estuarinos (53.99\%), tem um hábito demersal (35.92\%) e comportamento oceânico migratório (28.87\%). A maioria dos registros diz respeito a peixes carnívoros $(72.54 \%)$ e com algum valor comercial (96.48\%). A comparação da lista de ictiofauna registrada para os sambaquis com uma lista construída com base em inventários atuais de peixes para mesma região não mostrou diferenças significativas (teste de qui-quadrado, $\mathrm{p}>0.99$ ). Dessa forma, os resultados apresentados indicam que os vestígios de ictiofauna encontrados em sambaquis constituem uma amostra relevante da biodiversidade do Holoceno podendo ser muito úteis na construção de inventários de referências mais completos da fauna ictiológica da costa brasileira.

Palavras-chave: Ictiofauna, Inventário de referências, Pescadores-Coletores-Caçadores, Riqueza de espécies, Sambaquis, Zooarqueologia
\end{abstract}

\section{Introduction}

Biodiversity-related research has developed significantly since the 1990s, when ecologists worldwide, concerned with anthropogenic effects on ecosystems, intensified their studies of environmental issues (Amaral \& Jablonsky 2005, Lewinsohn \& Prado 2005). Biodiversity is defined by the Convention on Biological Diversity as the variability among living beings of all origins, including terrestrial, marine and other aquatic ecosystems, and their associated ecological complexes, including intra- and inter-species as well as ecosystem diversity (Arruda et al. 2000).
Recently, the Census of Marine Life raised the estimated number of known marine species from approximately 230,000 to between 1 and 1.4 million; more than 1,200 new species were identified among specimens collected in known and previously unexplored waters (Costello et al. 2010). Furthermore, in a review of research related to Brazilian biodiversity, Siqueira et al. (2015) found that only $21 \%$ of 1,156 references from 2009 to 2014 addressed the richness of aquatic species, indicating that the marine environment remains little studied and is therefore largely unknown. Finally, baselines for long-term studies of marine biodiversity are scarce (Knowlton \& Jackson 2008, Pinnegar \& Engelhard 2008). 
Baselines are reference biodiversity inventories that directly assess the species composition of a specific site for a given spatial extent and time. The data generated by such inventories are one of the most important tools for the conservation and management of natural areas, especially the associated endangered species (Silveira et al. 2010). The establishment of baselines is particularly important for the conservation of marine fish because this group is intensely exploited due to their commercial value and account for a significant share of global fishery production. In Brazil, fisheries have been key to the development of the country, concentrating $70 \%$ of the population near the coast, and the sea plays a key role in its history, culture and economy (Rosa \& Lima 2008).

To be as accurate as possible, reference inventories of ichthyological fauna should include not only current data but also historic and prehistoric (fossil/sub-fossil) data as well (Furon 1969, Warwick \& Light 2002, Willis \& Birks 2006, Froyd \& Willis 2008, Stahl 2008). However, collecting data on the species compositions of the past is complicated because fossil/sub-fossil records are largely characterised by being incomplete; that is, the biological data (species morphology, richness, diversity and evenness, among others) preserved in those records are influenced by non-linear modifications that occur from the time of death to the final burial of an organism (Ritter \& Erthal 2016) and the species preservation potential (Prummel \& Heinrich 2005). Therefore, prehistoric records are relatively scarce (Bittencourt et al. 2015).

However, there are some Holocene species composition records from sources including beaches, death assemblages and middens. Beaches and restingas originated in the Holocene, but their characteristics prevent the establishment of a chronology (Lacerda et al. 1984). Conversely, death assemblages allow for accurate chronological estimates and have the great advantage of being natural, thus showing actual tanatocenoses, but these formations are rare along the Brazilian coast (Ritter \& Erthal 2016). However, middens, which are archaeological sites dating between 8,000 and 1,000 years before present (BP, according to the convention before 1950), are commonly found on the Brazilian coast and allow a chronology to be established because they show a stratigraphic sequence of different species compositions (Kneip et al. 1988, Gaspar 1998, Scheel-Ybert et al. 2009, Klokler et al. 2010).

Middens were built by groups of prehistoric fishermen-gatherers-hunters, which explains why they are found in estuarine areas at the intersections of rivers and the sea. These sites contain a wealth of resources including sediments, coal, lithic material and, above all, faunal remains. High numbers of molluscs and crustaceans, including sea and sand crabs, as well as echinoderms and fish have been found among the marine zoo-archaeological remains recovered from middens (DeBlasis et al. 2007, Figuti 1993, Lima 2000, Lima et al. 2003).

For fish species, their zoo-archaeological remains indicate their usefulness to midden populations, so most species recorded at these sites have a cultural meaning and show sociocultural and identity relationships and spatiotemporal economic characteristics (Figuti 1998, Barbosa-Guimarães 2013, Wagner \& Silva 2014, Lopes et al. 2016). Accordingly, because the ichthyological remains in middens represent the diversity of prehistoric fishes obtained from a selective catch, this data source underestimates the diversity of Holocene fish. That is, the species diversity of the remains found in the middens is lower than that in natural communities (Gonzalez 2005, Costa et al. 2012).

Although midden fish records underestimate natural diversity, they are a key source of information on the ichthyological fauna of the past because prehistoric people could only have caught the fish available in the environments at the time. Furthermore, by-catch occurred; that is, species with no known anthropological relevance were incidentally fished with target species (Reitz \& Wing 2008, Villagran \& Gianini 2014, Beuclair et al. 2016). Therefore, midden records are a good indicator of Holocene biodiversity, providing data on fish species composition, abundance, distribution and richness as well as cultural information (diet, fishing gear, ritualistic symbols, ornaments and artefacts) (Souza et al. 2010a, Souza et al. 2010b, Faria et al. 2014, Mendes et al. 2014, Rodrigues et al. 2016a, Rodrigues et al. 2016b, Beuclair et al. 2016, Silva et al. 2016).

Thus, although middens are artificial accumulations (that is, built by prehistoric populations) of biological material, the presence or absence of species at these sites provides sufficient data to create a taxonomic list that may be useful for defining a historical record of biological diversity (Stahl 2008), so this study presents an inventory of marine ichthyological biodiversity from southeast Brazilian middens. This list is the first comprehensive inventory of Holocene fish fauna from this region and may help elucidate past natural ocean events, enabling the establishment of more complete baselines to inform conservation and management measures.

\section{Methods}

The inventory was constructed from an extensive bibliographical survey of the libraries of universities and institutions with archaeological collections from sites along the southeast coast of Brazil as well as online databases (Web of Knowledge, Scientific Electronic Library Online - SciELO, Google Scholar and the Thesis Database of the Brazilian Federal Agency for the Support and Evaluation of Graduate Education (Coordenação de Aperfeiçoamento de Pessoal de Nível Superior CAPES)). The data sources included scientific articles and books, thesis, dissertations, monographs and technical reports.

The list of species was analysed in terms of absolute and relative species richness and absolute and relative frequencies of occurrence. Absolute species richness is the number of species present at a sampled site, while the relative species richness is the ratio between that number and the total number of species. Similarly, the absolute frequency of occurrence is the number of sites at which a specific species occurs, and the relative frequency is the ratio between that number and the total number of sites.

Furthermore, taxonomic, biogeographic, ecological and economic data on the inventoried species were collected from the FishBase database (Froese \& Pauly 2017) and categorised by distribution (range of occurrence), environment (habitat), habit (way of life at specific locations in the water column), behaviour (migratory movements), feeding guild (food requirements) and commercial value (demand for the species in the fish market).

The checklists constructed by Bizerril \& Costa (2001) for Rio de Janeiro and Menezes (2011) for São Paulo were compared along with the inventory from the midden zoo-archaeological remains from this study to current ichthyological inventories for the same regions, and the species catalogued in speciesLink, a digital information system that integrates primary data from scientific collections in real time, for Espírito Santo were surveyed. Furthermore, a chi-squared test $\left(\chi^{2}\right)$ was performed to assess whether the ratio of commercial and non-commercial species in the midden fish inventory differed significantly from the ratios in current fish checklists.

\section{Results}

Data were collected from 68 middens distributed in 19 locations along the coast of three states of the Southeast Region of Brazil: Espírito Santo (ES), Rio de Janeiro (RJ) and São Paulo (SP) (Figure 1). The location with the highest number of middens (12 sites, 17.65\%) was Paraty in the state of Rio de Janeiro, which also had the highest number of documented sites $(53,77.95 \%)$. Conversely, Espírito Santo had the lowest number of 
middens (2, 2.94\%; Table 1). From these total of 68 middens, 49 of them had records of ${ }^{14} \mathrm{C}$ radiocarbonic dates (Figure 2). Occupation time ranged from 8,182 BP (Sambaqui de Camboinhas) to 675 BP (Sítio do Major). However, the majority of them were built and occupied during the period defined by Walker et al. (2012) as Late Holocene.

A total of 142 fish species were inventoried, and most taxa belonged to class Osteichthyes $(105,73.94 \%)$. Sciaenidae was the most represented family with 21 species, and Cynoscion Gill, 1861 was the genus with the highest number of species, seven in total (Table 2). Of the Chondrichthyes $(37,26.06 \%)$, the family with the highest number of species was Carcharhinidae with 17 species, and the most representative genus was Carcharhinus Blainville, 1816 with 12 species (Table 3).

The site with the highest species richness was the midden Sambaqui do Algodão in Angra dos Reis with 71 inventoried species and a relative species richness value of 0.5 (Table 1). Most of the inventoried species $(63.38 \%)$ may be considered rare because they were recorded in no more than five of the 68 study sites. Conversely, Micropogonias furnieri (Desmarest, 1823) and Pogonias cromis (Linnaeus, 1766) had the highest frequencies of occurrence, being found in 53 (relative frequency $=0.78$ ) and 48 (relative frequency $=0.71$ ) of the sites, respectively.
The biogeographic, ecological and economic data showed that most of the identified fish are widely distributed in the Western Atlantic (59.72\%) and inhabit estuarine environments (53.99\%), while most species have a demersal habit (35.92\%) and exhibit oceanic migratory behaviour (28.87\%). Lastly, the surveyed fish are predominantly carnivorous (72.54\%) with some commercial value (96.48\%; Figure 3$)$.

In comparison to the current fish checklists, the middens contained $17.60 \%$ of the species recorded in Rio de Janeiro, $15.57 \%$ of the species recorded in São Paulo and 2.13\% of the species recorded in Espírito Santo. Overall, $17.57 \%$ of the fish recorded in the entire Southeast Region were represented in the middens, but they accounted for $28.07 \%$ of the species in the region with some commercial value. Moreover, exclusively historic species were identified including 13 in Rio de Janeiro, 15 in São Paulo, and four in Espírito Santo (Table 4). The results from the $\chi^{2}$ tests indicated that were no significant differences in the number of commercial species in the historic and current inventories for the states (Rio de Janeiro: $\chi^{2}=4.587 \times 10^{-9}$, degrees of freedom $=3$, probability $>0.995$; São Paulo: $\chi^{2}=3.549 \times 10^{-12}$, degrees of freedom $=3$, probability $>0.995$; Espírito Santo: $\chi^{2}=0.106$, degrees of freedom $=3$, probability $>0.99$ ) or the entire Southeast Region $\left(\chi^{2}=6.349 \times 10^{-17}\right.$, degrees of freedom $=3$, probability $\left.>0.995\right)$.

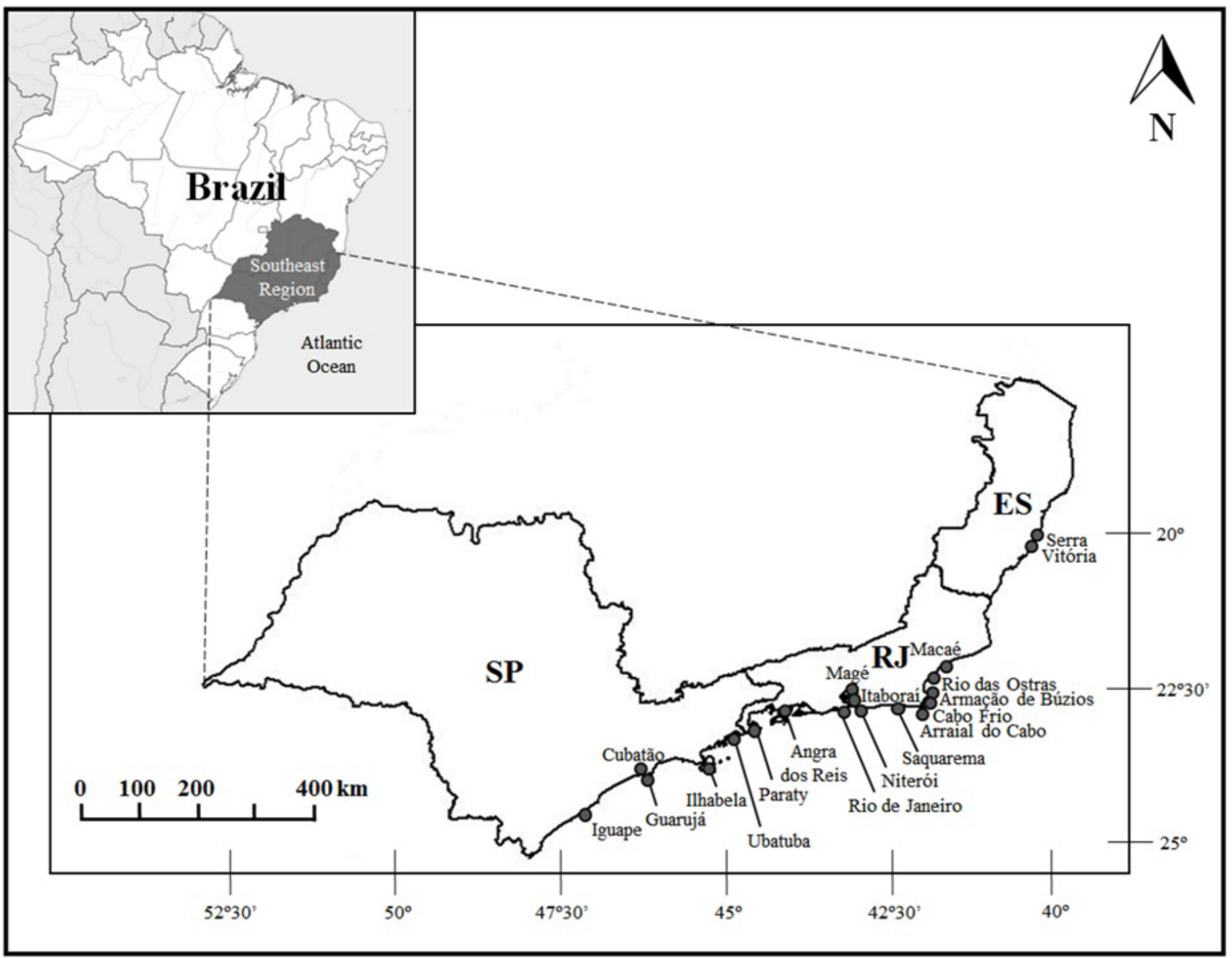

Figure 1. Map of the Southeast Region of Brazil with the locations of the inventoried middens. 
Mendes, A.B. et al.

Table 1. Middens inventoried in the Southeast Region of Brazil with absolute (Rspp) and relative (relative Rspp) species richness and the associated references from the literature.

\begin{tabular}{|c|c|c|c|c|c|}
\hline Location & Midden & Code & Rspp & Relative Rspp & $\begin{array}{r}\text { References } \\
\end{array}$ \\
\hline \multirow{8}{*}{$\begin{array}{l}\text { Angra dos } \\
\quad \text { Reis }\end{array}$} & Sambaqui da Caieira & 1 & 27 & 0.190 & $\begin{array}{l}\text { Lima 1991, DSc Thesis, FFLCH-USP } \\
\text { Lopes et al. 2016, PLoS One 11(6): 1-36 }\end{array}$ \\
\hline & $\begin{array}{l}\text { Sambaqui da Caieira } \\
\text { II }\end{array}$ & 2 & 29 & 0.204 & $\begin{array}{l}\text { Lima 1991, DSc Thesis, FFLCH-USP } \\
\text { Figuti 1998, Revista Arqueologia 11: 57-70 }\end{array}$ \\
\hline & $\begin{array}{l}\text { Sambaqui do } \\
\text { Algodão }\end{array}$ & 3 & 71 & 0.500 & $\begin{array}{l}\text { Lima 1991, DSc Thesis, FFLCH-USP } \\
\text { Lopes et al. 2016, PLoS One 11(6): 1-36 } \\
\text { Figuti 1998, Revista Arqueologia 11: } 57-70\end{array}$ \\
\hline & Sítio do Bigode I & 4 & 38 & 0.268 & $\begin{array}{l}\text { Lima 1991, DSc Thesis, FFLCH-USP } \\
\text { Lopes et al. 2016, PLoS One 11(6): 1-36 } \\
\text { Figuti 1998, Revista Arqueologia 11: } 57-70\end{array}$ \\
\hline & Sítio do Major & 5 & 47 & 0.331 & $\begin{array}{l}\text { Lima 1991, DSc Thesis, FFLCH-USP } \\
\text { Lopes et al. 2016, PLoS One 11(6): 1-36 } \\
\text { Figuti 1998, Revista Arqueologia 11: } 57-70\end{array}$ \\
\hline & Sítio do Peri & 6 & 39 & 0.275 & $\begin{array}{l}\text { Lima 1991, DSc Thesis, FFLCH-USP } \\
\text { Lopes et al. 2016, PLoS One 11(6): 1-36 } \\
\text { Figuti 1998, Revista Arqueologia 11: 57-70 }\end{array}$ \\
\hline & Sítio Ilhota do Leste & 7 & 14 & 0.099 & $\begin{array}{l}\text { Lima 1991, DSc Thesis, FFLCH-USP } \\
\text { Lessa \& Carvalho 2015, Bol Mus Para Emílio Goeldi 10: 489-507 } \\
\text { Tenório 2003, DSc Thesis, PUC-RS } \\
\text { Tenório 2003, Anais XII Congresso da SAB } \\
\text { Lessa \& Coelho 2010, Rev Mus Arqueol Etnol 20: 77-89 }\end{array}$ \\
\hline & Sambaqui do Acaiá & 8 & 23 & 0.162 & Lopes et al. 2016, PLoS One 11(6): 1-36 \\
\hline Macaé & $\begin{array}{l}\text { Sítio da Ilha de } \\
\text { Santana }\end{array}$ & 9 & 13 & 0.092 & Lima 1991, DSc Thesis, FFLCH-USP \\
\hline \multirow{4}{*}{ Magé } & Sítio Saracuruna & 10 & 5 & 0.035 & $\begin{array}{l}\text { Lima 1991, DSc Thesis, FFLCH-USP } \\
\text { Mendonça de Souza 1981, Pré-história Fluminense, Instituto Estadual do } \\
\text { Patrimônio Cultural, RJ } \\
\text { Mello \& Mendonça de Souza 1977, Nheengatu 1: 43-58 }\end{array}$ \\
\hline & $\begin{array}{l}\text { Sambaqui Rio das } \\
\text { Pedrinhas }\end{array}$ & 11 & 9 & 0.063 & $\begin{array}{l}\text { Mendonça de Souza \& Mendonça de Souza 1981-1982, Arquivos do } \\
\text { Museu de História Natural-UFMG 6/7: 109-131 }\end{array}$ \\
\hline & $\begin{array}{l}\text { Sambaqui de } \\
\text { Sernambetiba }\end{array}$ & 12 & 2 & 0.014 & $\begin{array}{l}\text { Beltão et al. 1981-1982, Arquivos do Museu de História Natural-UFMG } \\
\text { 6/7: 145-155 } \\
\text { Perez et al. } 1995 \text { In: Arqueologia do Estado do Rio de Janeiro, Arquivo } \\
\text { Público do Estado do RJ pp. 29-34 }\end{array}$ \\
\hline & $\begin{array}{l}\text { Sambaqui de } \\
\text { Amourins }\end{array}$ & 13 & 1 & 0.007 & $\begin{array}{l}\text { Heredia et al. 1981-1982, Arquivos do Museu de História Natural-UFMG } \\
\text { 6/7: 175-188 }\end{array}$ \\
\hline \multirow{12}{*}{ Paraty } & $\begin{array}{l}\text { Abrigo Ponta do } \\
\text { Leste I }\end{array}$ & 14 & 5 & 0.035 & $\begin{array}{l}\text { Mendonça de Souza \& Mendonça de Souza 1981-1982, Arquivos do } \\
\text { Museu de História Natural-UFMG 6/7: 109-131 }\end{array}$ \\
\hline & $\begin{array}{l}\text { Abrigo Ponta do } \\
\text { Leste II }\end{array}$ & 15 & 5 & 0.035 & $\begin{array}{l}\text { Mendonça de Souza 1981, Pré-história Fluminense, Instituto Estadual do } \\
\text { Patrimônio Cultural, RJ }\end{array}$ \\
\hline & $\begin{array}{l}\text { Sambaqui Olho } \\
\text { D’Água }\end{array}$ & 16 & 3 & 0.021 & $\begin{array}{l}\text { Mendonça de Souza 1981, Pré-história Fluminense, Instituto Estadual do } \\
\text { Patrimônio Cultural, RJ } \\
\text { Mendonça de Souza \& Mendonça de Souza 1981-1982, Arquivos do } \\
\text { Museu de História Natural-UFMG 6/7: 109-131 }\end{array}$ \\
\hline & Toca do Cassununga & 17 & 5 & 0.035 & $\begin{array}{l}\text { Mendonça de Souza 1981, Pré-história Fluminense, Instituto Estadual do } \\
\text { Patrimônio Cultural, RJ }\end{array}$ \\
\hline & Toca dos Caboclos I & 18 & 5 & 0.035 & $\begin{array}{l}\text { Mendonça de Souza \& Mendonça de Souza 1981-1982, Arquivos do } \\
\text { Museu de História Natural-UFMG 6/7: 109-131 }\end{array}$ \\
\hline & $\begin{array}{l}\text { Abrigo da Ilha } \\
\text { Pelada }\end{array}$ & 19 & 5 & 0.035 & $\begin{array}{l}\text { Mendonça de Souza \& Mendonça de Souza 1981-1982, Arquivos do } \\
\text { Museu de História Natural-UFMG 6/7: 109-131 }\end{array}$ \\
\hline & Sítio Trindade III & 20 & 6 & 0.042 & $\begin{array}{l}\text { Mendonça de Souza 1981, Pré-história Fluminense, Instituto Estadual do } \\
\text { Patrimônio Cultural, RJ }\end{array}$ \\
\hline & Sambaqui do Forte & 21 & 5 & 0.035 & $\begin{array}{l}\text { Mendonça de Souza \& Mendonça de Souza 1981-1982, Arquivos do } \\
\text { Museu de História Natural-UFMG 6/7: 109-131 }\end{array}$ \\
\hline & $\begin{array}{l}\text { Sambaqui do } \\
\text { Perequê-Açu }\end{array}$ & 22 & 5 & 0.035 & $\begin{array}{l}\text { Mendonça de Souza \& Mendonça de Souza 1981-1982, Arquivos do } \\
\text { Museu de História Natural-UFMG 6/7: 109-131 }\end{array}$ \\
\hline & $\begin{array}{l}\text { Sambaqui da Ilha } \\
\text { Comprida }\end{array}$ & 23 & 5 & 0.035 & $\begin{array}{l}\text { Mendonça de Souza \& Mendonça de Souza 1981-1982, Arquivos do } \\
\text { Museu de História Natural-UFMG 6/7: 109-131 }\end{array}$ \\
\hline & Sambaqui do Pouso & 24 & 5 & 0.035 & $\begin{array}{l}\text { Mendonça de Souza \& Mendonça de Souza 1981-1982, Arquivos do } \\
\text { Museu de História Natural-UFMG 6/7: 109-131 }\end{array}$ \\
\hline & $\begin{array}{l}\text { Sambaqui de } \\
\text { Mamanguá }\end{array}$ & 25 & 5 & 0.035 & $\begin{array}{l}\text { Mendonça de Souza \& Mendonça de Souza 1981-1982, Arquivos do } \\
\text { Museu de História Natural-UFMG 6/7: 109-131 }\end{array}$ \\
\hline
\end{tabular}


Table 1. Continued...

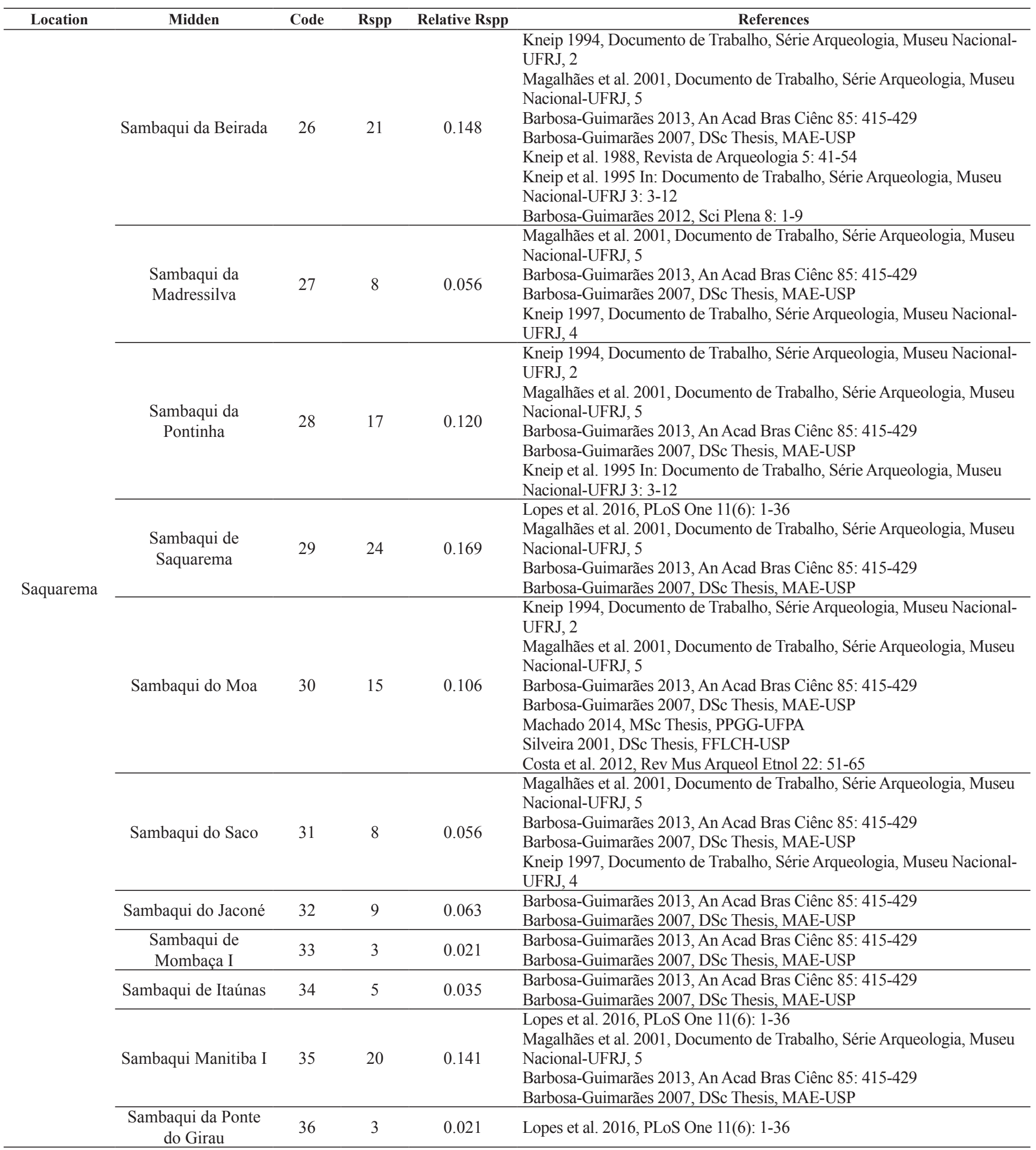


Table 1. Continued...

\begin{tabular}{|c|c|c|c|c|c|}
\hline Location & Midden & Code & Rspp & Relative Rspp & References \\
\hline \multirow{6}{*}{ Cabo Frio } & Sambaqui do Forte & 37 & 2 & 0.014 & $\begin{array}{l}\text { Mendonça de Souza \& Mendonça de Souza 1981-1982, Arquivos do } \\
\text { Museu de História Natural-UFMG 6/7: 109-131 } \\
\text { Costa et al. 2012, Rev Mus Arqueol Etnol 22: 51-65 } \\
\text { Kneip 1980, Separata de Pesquisas, Série Antropologia, } 31 \\
\text { Kneip et al. 1975, Separata da Revista do Museu Paulista-USP } \\
\text { Kneip et al. 1975, An Acad Bras Ciênc 47: 91-97 } \\
\text { Gaspar 1991, DSc Thesis, FFLCH-USP } \\
\text { Gaspar 2003, Pesquisas, Série Antropologia-IAP 59: 1-163 } \\
\text { Souza 2009, Monography, GeoQuater-UFRJ }\end{array}$ \\
\hline & $\begin{array}{l}\text { Abrigo Arraial do } \\
\text { Cabo }\end{array}$ & 38 & 5 & 0.035 & $\begin{array}{l}\text { Mendonça de Souza \& Mendonça de Souza 1981-1982, Arquivos do } \\
\text { Museu de História Natural-UFMG 6/7: 109-131 }\end{array}$ \\
\hline & $\begin{array}{l}\text { Sambaqui Boca da } \\
\text { Barra }\end{array}$ & 39 & 10 & 0.070 & $\begin{array}{l}\text { Gaspar 1991, DSc Thesis, FFLCH-USP } \\
\text { Gaspar 2003, Pesquisas, Série Antropologia-IAP 59: 1-163 } \\
\text { Barbosa 1999, MSc Thesis, PGCA-UFF }\end{array}$ \\
\hline & Sítio do Meio & 40 & 1 & 0.007 & $\begin{array}{l}\text { Gaspar 1991, DSc Thesis, FFLCH-USP } \\
\text { Gaspar 2003, Pesquisas, Série Antropologia-IAP 59: 1-163 } \\
\text { Gaspar \& Scaramella 1992, Anais VI Reunião Científica da SAB }\end{array}$ \\
\hline & $\begin{array}{l}\text { Sambaqui da Salina } \\
\text { Peroano }\end{array}$ & 41 & 2 & 0.014 & $\begin{array}{l}\text { Gaspar 1991, DSc Thesis, FFLCH-USP } \\
\text { Gaspar 2003, Pesquisas, Série Antropologia-IAP 59: 1-163 } \\
\text { Franco \& Gaspar 1992, Anais VI Reunião Científica da SAB }\end{array}$ \\
\hline & $\begin{array}{l}\text { Sítio Ilha de Cabo } \\
\text { Frio }\end{array}$ & 42 & 15 & 0.106 & Lopes et al. 2016, PLoS One 11(6): 1-36 \\
\hline $\begin{array}{l}\text { Armação de } \\
\text { Búzios }\end{array}$ & Geribá II & 43 & 1 & 0.007 & $\begin{array}{l}\text { Gaspar 1991, DSc Thesis, FFLCH-USP } \\
\text { Gaspar 2003, Pesquisas, Série Antropologia-IAP 59: 1-163 }\end{array}$ \\
\hline \multirow{4}{*}{$\begin{array}{l}\text { Arraial do } \\
\text { Cabo }\end{array}$} & $\begin{array}{l}\text { Sítio Colônia de } \\
\text { Pesca }\end{array}$ & 44 & 2 & 0.014 & $\begin{array}{l}\text { Gaspar 1991, DSc Thesis, FFLCH-USP } \\
\text { Mendonça de Souza et al. 1983-1984, Arquivos do Museu de História } \\
\text { Natural-UFMG 8/9: 107-119 } \\
\text { Tenório et al. 2010, Rev Mus Arqueol Etnol 20: 127-145 } \\
\text { Tenório et al. 2005, Anais XIII Congresso da SAB }\end{array}$ \\
\hline & $\begin{array}{l}\text { Sítio Ponta da } \\
\text { Cabeça }\end{array}$ & 45 & 9 & 0.063 & $\begin{array}{l}\text { Gaspar 1991, DSc Thesis, FFLCH-USP } \\
\text { Tenório et al. 2010, Rev Mus Arqueol Etnol 20: 127-145 } \\
\text { Tenório et al. 2005, Anais XIII Congresso da SAB }\end{array}$ \\
\hline & $\begin{array}{l}\text { Sítío do Condomínio } \\
\text { do Atalaia }\end{array}$ & 46 & 1 & 0.007 & Tenório et al. 2005, Anais XIII Congresso da SAB \\
\hline & Usiminas & 47 & 24 & 0.169 & Lopes et al. 2016, PLoS One 11(6): 1-36 \\
\hline Mangaratiba & Sambaqui do Saí & 48 & 5 & 0.035 & $\begin{array}{l}\text { Mendonça de Souza \& Mendonça de Souza 1981-1982, Arquivos do } \\
\text { Museu de História Natural-UFMG 6/7: 109-131 }\end{array}$ \\
\hline Rio das Ostras & Sambaqui da Tarioba & 49 & 15 & 0.106 & Tuna 2015, MSc Thesis, PBMAC-UFF \\
\hline Niterói & $\begin{array}{l}\text { Sambaqui de } \\
\text { Camboinhas }\end{array}$ & 50 & 12 & 0.085 & $\begin{array}{l}\text { Lopes et al. 2016, PLoS One 11(6): 1-36 } \\
\text { Souza Cunha et al. } 1981 \text { In: Pesquisas arqueológicas no litoral de Itaipu, } \\
\text { Niterói, RJ, Cia de Desenvolvimento Territorial } \\
\text { Souza Cunha et al. 1986, Anais XXX Congresso Brasileiro de Geologia } \\
\text { Vogel \& Veríssimo } 1981 \text { In: Pesquisas arqueológicas no litoral de Itaipu, } \\
\text { Niterói, RJ, Cia de Desenvolvimento Territoria } \\
\text { Kneip \& Pallestrini } 1984 \text { In: Restigas: origem, estrutura, processo, CEUFF } \\
\text { Kneip 1979, Pesquisas de salvamento em Itaipu, Niterói, Rio de Janeiro, } \\
\text { Cia de Desenvolvimento Territorial } \\
\text { Kneip 1995, Documento de Trabalho, Série Arqueologia, Museu Nacional- } \\
\text { UFRJ 2: 83-102 }\end{array}$ \\
\hline Itaboraí & Sambaqui Sampaio I & 51 & 1 & 0.007 & Pinto 2009, MSc Thesis, PPGArq-UFRJ \\
\hline \multirow[b]{2}{*}{ Rio de Janeiro } & $\begin{array}{l}\text { Sambaqui da } \\
\text { Embratel }\end{array}$ & 52 & 2 & 0.014 & $\begin{array}{l}\text { Kneip et al. } 1984 \text { In: Restigas: origem, estrutura, processo, CEUFF } \\
\text { Kneip et al. 1984, Revista de Pré-História 6: } 334-360\end{array}$ \\
\hline & $\begin{array}{l}\text { Sambaqui do Zé } \\
\text { Espinho }\end{array}$ & 53 & 12 & 0.085 & $\begin{array}{l}\text { Kneip et al. } 1984 \text { In: Restigas: origem, estrutura, processo, CEUFF } \\
\text { Kneip et al. 1984, Revista de Pré-História 6: } 334-360 \\
\text { Vogel } 1987 \text { In: Coletores e pescadores pré-históricos de Guaratiba, Rio de } \\
\text { Janeiro, EDUFF } \\
\text { Kneip et al. 1986, Separata da Rev Mus Pau }\end{array}$ \\
\hline
\end{tabular}


Table 1. Continued...

\begin{tabular}{|c|c|c|c|c|c|}
\hline Location & Midden & Code & Rspp & Relative Rspp & References \\
\hline \multirow{5}{*}{ Cubatão } & $\begin{array}{l}\text { Sambaqui } \\
\text { Piaçaguera }\end{array}$ & 54 & 63 & 0.444 & $\begin{array}{l}\text { Fischer 2012, MSc Thesis, MAE-USP } \\
\text { Gonzalez 2005, DSc Thesis, MAE-USP } \\
\text { Gonzalez \& Amenomori 2003, Rev Mus Arqueol Etnol 13: 25-37 } \\
\text { Filippini \& Eggers 2005-2006, Rev Mus Arqueol Etnol 5-16: 165-180 } \\
\text { Garcia 1972, DSc Thesis, IB-USP } \\
\text { Garcia \& Uchôa 1980, Revista de Pré-História 2: 5-81 } \\
\text { Duarte 1968, O sambaqui visto através de alguns sambaquis, Instituto de } \\
\text { Pré-História da USP } \\
\text { Borges 2015, DSc Thesis, Muséum National D’Histoire Naturelle de Paris }\end{array}$ \\
\hline & COSIPA 1 & 55 & 35 & 0.246 & $\begin{array}{l}\text { Figuti 1998, Revista Arqueologia 11: 57-70 } \\
\text { Gonzalez 2005, DSc Thesis, MAE-USP } \\
\text { Figuti 1993, Rev Mus Arqueol Etnol 3: 67-80 } \\
\text { Figuti 1994-1995, Revista de Arqueologia 8: 267-283 }\end{array}$ \\
\hline & COSIPA 2 & 56 & 16 & 0.113 & $\begin{array}{l}\text { Gonzalez 2005, DSc Thesis, MAE-USP } \\
\text { Gonzalez \& Amenomori 2003, Rev Mus Arqueol Etnol 13: 25-37 } \\
\text { Figuti 1993, Rev Mus Arqueol Etnol 3: 67-80 } \\
\text { Figuti 1994-1995, Revista de Arqueologia 8: 267-283 }\end{array}$ \\
\hline & COSIPA 3 & 57 & 9 & 0.063 & $\begin{array}{l}\text { Figuti 1987, Institut de Paleontologie Humaine, Paris } \\
\text { Figuti 1989, Revista de Pré-História 7: 112-126 }\end{array}$ \\
\hline & COSIPA 4 & 58 & 36 & 0.254 & $\begin{array}{l}\text { Figuti 1998, Revista Arqueologia 11: 57-70 } \\
\text { Gonzalez 2005, DSc Thesis, MAE-USP } \\
\text { Figuti 1993, Rev Mus Arqueol Etnol 3: 67-80 } \\
\text { Figuti 1994-1995, Revista de Arqueologia 8: 267-283 }\end{array}$ \\
\hline \multirow{3}{*}{ Ubatuba } & Sítio Tenório & 59 & 38 & 0.268 & $\begin{array}{l}\text { Figuti 1998, Revista Arqueologia 11: 57-70 } \\
\text { Gonzalez 2005, DSc Thesis, MAE-USP } \\
\text { Gonzalez \& Amenomori 2003, Rev Mus Arqueol Etnol 13: 25-37 } \\
\text { Filippini \& Eggers 2005-2006, Rev Mus Arqueol Etnol 5-16: 165-180 } \\
\text { Garcia 1972, DSc Thesis, IB-USP } \\
\text { Amenomori 1999, MSc Thesis, FFLCH-USP }\end{array}$ \\
\hline & Sítio do Mar Virado & 60 & 28 & 0.197 & $\begin{array}{l}\text { Gonzalez \& Amenomori 2003, Rev Mus Arqueol Etnol 13: 25-37 } \\
\text { Amenomori 1999, MSc Thesis, FFLCH-USP } \\
\text { Amenomori 2005, DSc Thesis, MAE-USP } \\
\text { Uchôa 2009, CLIO-Série Arqueológica 24: 7-40 } \\
\text { Nishida 2001, MSc Thesis, MAE-USP }\end{array}$ \\
\hline & Sítio Couves 1 & 61 & 6 & 0.042 & Amenomori 1999, MSc Thesis, FFLCH-USP \\
\hline \multirow{3}{*}{ Guarujá } & $\begin{array}{l}\text { Sambaqui do } \\
\text { Buracão }\end{array}$ & 62 & 54 & 0.380 & $\begin{array}{l}\text { Gonzalez \& Amenomori 2003, Rev Mus Arqueol Etnol 13: 25-37 } \\
\text { Borges 2015, DSc Thesis, Muséum National D’Histoire Naturelle de Paris } \\
\text { Duarte 1968, O sambaqui visto através de alguns sambaquis, Instituto de } \\
\text { Pré-História da USP } \\
\text { Amenomori 2005, DSc Thesis, MAE-USP }\end{array}$ \\
\hline & $\begin{array}{c}\text { Sambaqui do Mar } \\
\text { Casado }\end{array}$ & 63 & 48 & 0.338 & $\begin{array}{l}\text { Gonzalez 2005, DSc Thesis, MAE-USP } \\
\text { Gonzalez \& Amenomori 2003, Rev Mus Arqueol Etnol 13: 25-37 } \\
\text { Borges 2015, DSc Thesis, Muséum National D’Histoire Naturelle de Paris } \\
\text { Duarte 1968, O sambaqui visto através de alguns sambaquis, Instituto de } \\
\text { Pré-História da USP }\end{array}$ \\
\hline & Sambaqui Maratuá & 64 & 24 & 0.169 & Gonzalez 2005, DSc Thesis, MAE-USP \\
\hline Ilhabela & $\begin{array}{l}\text { Sambaqui Abrigo } \\
\text { Sul }\end{array}$ & 65 & 2 & 0.014 & Bendazzoli 2014, DSc Thesis, MAE-USP \\
\hline Iguape & Abrigo do Pindú & 66 & 2 & 0.014 & $\begin{array}{l}\text { Bendazzoli 2014, DSc Thesis, MAE-USP } \\
\text { Bonetti 1997, MSc Thesis, FFLCH-USP }\end{array}$ \\
\hline Serra & $\begin{array}{l}\text { Sambaqui do } \\
\text { Péricles II }\end{array}$ & 67 & 4 & 0.028 & $\begin{array}{l}\text { Rhea Estudos e Projetos Ltda 2009, Salvamento e monitoramento } \\
\text { arqueológico na área do Alphaville Jacuhy }\end{array}$ \\
\hline Vitória & Sítio Campus 2 & 68 & 3 & 0.021 & Perota 1972, Revista de Cultura da UFES 3: 39-45 \\
\hline
\end{tabular}


Mendes, A.B. et al.

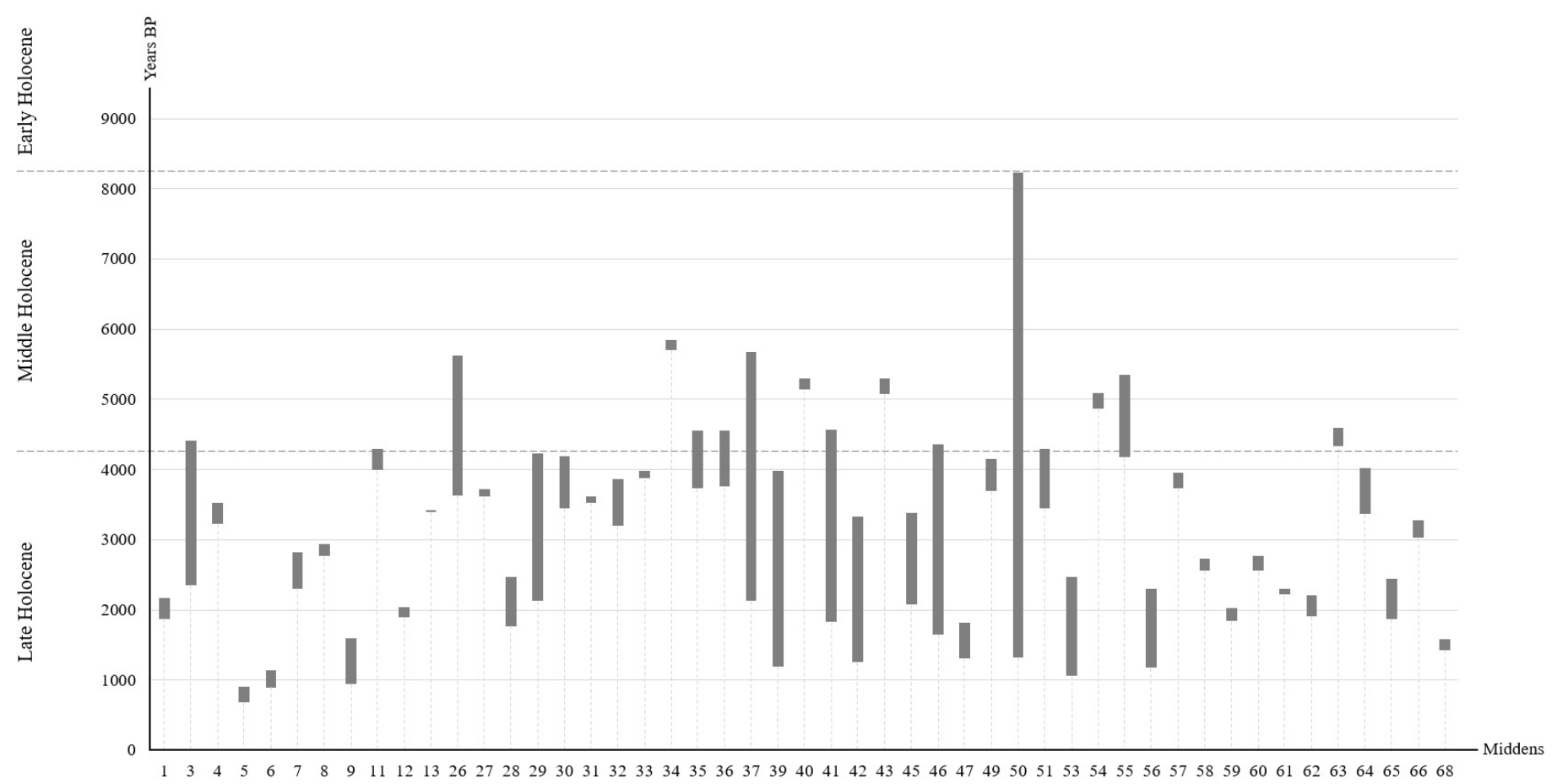

Figure 2. Graphic with the ${ }^{14} \mathrm{C}$ radiocarbonic dates of the 49 inventoried middens of the Southeast Region of Brazil. In the $\mathrm{x}$ axis are the middens (codes defined at Table 1) and in the $\mathrm{y}$ axis are the ranges of ${ }^{14} \mathrm{C}$ radiocarbonic dating (given in year before present) found in the literature. The different periods of the Holocene were defined based in Walker et al. (2012): Early Holocene (11,700 8,200 BP), Middle Holocene (8,200 4,200 BP) and Late Holocene (4,200 BP till today).

Table 2. Marine fish of class Osteichthyes from middens in the Southeast Region of Brazil with their absolute (F) and relative (relative F) frequencies of occurrence as well as the midden(s) in which they were recorded. The codes representing the middens are defined in Table 1.

\begin{tabular}{|c|c|c|c|c|}
\hline Family & $\begin{array}{c}\text { Species } \\
\end{array}$ & $\mathbf{F}$ & Relative F & Midden(s) \\
\hline Albulidae & Albula nemoptera (Fowler, 1911) & 1 & 0.015 & 3 \\
\hline \multirow{9}{*}{ Ariidae } & Aspistor luniscutis (Valenciennes, 1840) & 19 & 0.279 & $2,3,4,5,6,11,27,28,30,31,35,54,55,58,59,60,62,63,64$ \\
\hline & Bagre bagre (Linnaeus, 1766) & 23 & 0.338 & $1,2,3,4,5,6,9,10,11,20,21,22,23,24,25,48,50,54,55,58,59,62,63$ \\
\hline & Bagre marinus (Mitchill, 1815) & 18 & 0.265 & $1,2,3,4,5,6,8,26,28,30,35,49,53,54,62,63,64,67$ \\
\hline & Cathorops spixii (Agassiz, 1829) & 18 & 0.265 & $2,3,4,5,6,26,28,29,30,35,54,55,57,58,59,62,63,64$ \\
\hline & Genidens barbus (Lacepède, 1803) & 33 & 0.485 & $\begin{array}{c}2,3,4,5,6,10,11,12,20,21,22,23,24,25,26,27,28,29,30,31,32,33,34,35,48, \\
50,54,55,58,59,60,62,63\end{array}$ \\
\hline & Genidens genidens (Cuvier, 1829) & 19 & 0.279 & $2,3,4,5,6,8,26,28,29,35,49,50,54,55,58,59,62,63,64$ \\
\hline & Genidens machadoi (Miranda Ribeiro, 1918) & 1 & 0.015 & 54 \\
\hline & Notarius grandicassis (Valenciennes, 1840) & 12 & 0.176 & $2,3,4,5,6,54,55,58,59,62,63,64$ \\
\hline & Potamarius grandoculis (Steindachner, 1877) & 2 & 0.029 & 54,63 \\
\hline \multirow{2}{*}{ Balistidae } & Balistes capriscus Gmelin, 1789 & 2 & 0.029 & 62,63 \\
\hline & Balistes vetula Linnaeus, 1758 & 12 & 0.176 & $1,2,3,4,5,6,9,54,55,58,59,60$ \\
\hline \multirow{10}{*}{ Carangidae } & Caranx crysos (Mitchill, 1815) & 4 & 0.059 & $29,54,62,63$ \\
\hline & Caranx hippos (Linnaeus, 1766) & 23 & 0.338 & $1,2,3,4,5,6,8,9,26,27,28,29,30,31,35,47,50,53,54,62,63,64,67$ \\
\hline & Caranx ruber (Bloch, 1793) & 1 & 0.015 & $\begin{array}{l}62 \\
\end{array}$ \\
\hline & Chloroscombrus chrysurus (Linnaeus, 1766) & 1 & 0.015 & 62 \\
\hline & Oligoplites saurus (Bloch \& Schneider, 1801) & 4 & 0.059 & $8,26,28,30$ \\
\hline & Selar crumenophthalmus (Bloch, 1793) & 1 & 0.015 & 8 \\
\hline & Selene vomer (Linnaeus, 1758) & 3 & 0.044 & $8,47,62$ \\
\hline & Seriola dumerili (Risso, 1810) & 2 & 0.029 & 62,63 \\
\hline & Trachinotus falcatus (Linnaeus, 1758) & 1 & 0.015 & 65 \\
\hline & Trachurus lathami Nichols, 1920 & 1 & 0.015 & 54 \\
\hline \multirow{3}{*}{ Centropomidae } & Centropomus ensiferus Poey, 1860 & 3 & 0.044 & $3,5,42$ \\
\hline & Centropomus parallelus Poey, 1860 & 6 & 0.088 & $35,47,54,62,63,64$ \\
\hline & Centropomus undecimalis (Bloch, 1792) & 16 & 0.235 & $3,11,26,27,28,29,30,32,35,54,57,62,63,64,67,69$ \\
\hline Coryphaenidae & Coryphaena hippurus Linnaeus, 1758 & 1 & 0.015 & 8 \\
\hline Dactylopteridae & Dactylopterus volitans (Linnaeus, 1758) & 5 & 0.074 & $9,29,60,62,63$ \\
\hline \multirow{2}{*}{ Diodontidae } & Chilomycterus spinosus (Linnaeus, 1758) & 12 & 0.176 & $1,2,3,4,5,6,47,49,54,55,58,59$ \\
\hline & Diodon hystrix Linnaeus, 1758 & 11 & 0.162 & $2,3,4,5,6,9,54,55,58,59,63$ \\
\hline
\end{tabular}


Table 2. Continued...

\begin{tabular}{|c|c|c|c|c|}
\hline Family & Species & $\mathbf{F}$ & Relative F & Midden(s) \\
\hline \multirow{2}{*}{ Eleotridae } & Dormitator maculatus (Bloch, 1792) & 1 & 0.015 & 54 \\
\hline & Eleotris pisonis (Gmelin, 1789) & 1 & 0.015 & 62 \\
\hline Elopidae & Elops saurus Linnaeus, 1766 & 1 & 0.015 & 54 \\
\hline Ephippidae & Chaetodipterus faber (Broussonet, 1782) & 21 & 0.309 & $1,2,3,4,5,6,7,9,29,35,39,47,49,53,54,55,58,59,60,61,62$ \\
\hline \multirow{4}{*}{ Gerreidae } & Diapterus auratus Ranzani, 1842 & 2 & 0.029 & 3,5 \\
\hline & Diapterus rhombeus (Cuvier, 1829) & 5 & 0.074 & $3,5,6,54,62$ \\
\hline & Eugerres brasilianus (Cuvier, 1830) & 2 & 0.029 & 54,62 \\
\hline & Gerres cinereus (Walbaum, 1792) & 1 & 0.015 & 3 \\
\hline \multirow{9}{*}{ Haemulidae } & Anisotremus surinamensis (Bloch, 1791) & 3 & 0.044 & $54,62,63$ \\
\hline & Anisotremus virginicus (Linnaeus, 1758) & 6 & 0.088 & $1,3,4,5,6,47$ \\
\hline & Conodon nobilis (Linnaeus, 1758) & 13 & 0.191 & $2,3,4,5,6,11,54,55,58,59,60,62,63$ \\
\hline & Haemulon aurolineatum Cuvier, 1830 & 2 & 0.029 & 3,47 \\
\hline & Haemulon plumierii (Lacepède, 1801) & 3 & 0.044 & $54,62,63$ \\
\hline & Haemulon sciurus (Shaw, 1803) & 1 & 0.015 & 3 \\
\hline & Haemulon steindachneri (Jordan \& Gilbert, 1882) & 3 & 0.044 & $1,3,47$ \\
\hline & Orthopristis ruber (Cuvier, 1830) & 10 & 0.147 & $1,2,3,4,5,6,54,55,58,59$ \\
\hline & Pomadasys crocro (Cuvier, 1830) & 1 & 0.015 & 63 \\
\hline Hemiramphidae & Hyporhamphus unifasciatus (Ranzani, 1841) & 2 & 0.029 & 1,3 \\
\hline Holocentridae & Holocentrus adscensionis (Osbeck, 1765) & 2 & 0.029 & 60,62 \\
\hline Istiophoridae & Istiophorus albicans (Latreille, 1804) & 1 & 0.015 & 47 \\
\hline Kyphosidae & Kyphosus sectatrix (Linnaeus, 1758) & 3 & 0.044 & $9,49,60$ \\
\hline Labridae & Bodianus rufus (Linnaeus, 1758) & 4 & 0.059 & $3,8,42,47$ \\
\hline Lobotidae & Lobotes surinamensis (Bloch, 1790) & 10 & 0.147 & $7,39,54,55,56,58,62,63,64,66$ \\
\hline \multirow{5}{*}{ Lutjanidae } & Lutjanus analis (Cuvier, 1828) & 1 & 0.015 & 68 \\
\hline & Lutjanus griseus (Linnaeus, 1758) & 1 & 0.015 & 59 \\
\hline & Lutjanus purpureus (Poey, 1866) & 1 & 0.015 & 59 \\
\hline & Lutjanus synagris (Linnaeus, 1758) & 5 & 0.074 & $1,3,4,5,6$ \\
\hline & Ocyurus chrysurus (Bloch, 1791) & 1 & 0.015 & 47 \\
\hline Malacanthidae & Caulolatilus chrysops (Valenciennes, 1833) & 1 & 0.015 & 53 \\
\hline Megalopidae & Megalops atlanticus Valenciennes, 1847 & 3 & 0.044 & $54,62,63$ \\
\hline Mugilidae & Mugil liza Valenciennes, 1836 & 5 & 0.074 & $1,3,4,5,6$ \\
\hline Paralichthyidae & Paralichthys orbignyanus (Valenciennes, 1839) & 1 & 0.015 & 54 \\
\hline Polynemidae & Polydactylus oligodon (Günther, 1860) & 6 & 0.088 & $14,15,17,18,19,38$ \\
\hline Pomacanthidae & Pomacanthus paru (Bloch, 1787) & 1 & 0.015 & 62 \\
\hline Pomatomidae & Pomatomus saltatrix (Linnaeus, 1766) & 21 & 0.309 & $2,3,4,5,6,7,9,26,28,29,30,39,41,45,53,54,55,58,59,60,63$ \\
\hline \multirow{21}{*}{ Sciaenidae } & Bairdiella ronchus (Cuvier, 1830) & 13 & 0.191 & $1,2,3,4,5,6,32,49,54,55,57,58,59$ \\
\hline & Cynoscion acoupa (Lacepède, 1801) & 16 & 0.235 & $10,11,14,15,17,18,19,20,27,35,38,54,59,62,63,64$ \\
\hline & Cynoscion guatucupa (Cuvier, 1830) & 1 & 0.015 & 64 \\
\hline & Cynoscion jamaicensis (Vaillant \& Bocourt, 1883) & 12 & 0.176 & $1,2,3,4,5,6,8,32,34,49,55,58$ \\
\hline & Cynoscion leiarchus (Cuvier, 1830) & 5 & 0.074 & $54,55,58,62,63$ \\
\hline & Cynoscion similis Randall \& Cervigón, 1968 & 6 & 0.088 & $1,3,4,5,6,36$ \\
\hline & Cynoscion striatus (Cuvier, 1829) & 3 & 0.044 & $28,29,35$ \\
\hline & Cynoscion virescens (Cuvier, 1830) & 19 & 0.279 & $11,14,15,16,17,18,19,20,21,22,23,24,25,38,48,58,59,62,63$ \\
\hline & Isopisthus parvipinnis (Cuvier, 1830) & 3 & 0.044 & $32,54,59$ \\
\hline & Larimus breviceps Cuvier, 1830 & 18 & 0.265 & $1,2,3,4,5,6,32,33,34,49,54,55,57,58,59,60,61,63$ \\
\hline & Menticirrhus americanus (Linnaeus, 1758) & 2 & 0.029 & 49,59 \\
\hline & Micropogonias furnieri (Desmarest, 1823) & 53 & 0.779 & $\begin{array}{c}1,2,3,4,5,6,7,8,9,10,11,14,15,16,17,18,19,20,21,22,23,24,25,26,27,28 \\
29,30,31,32,33,34,35,36,38,39,48,49,50,51,52,53,54,55,56,57,58,59,60 \\
61,62,63,64\end{array}$ \\
\hline & Micropogonias undulatus (Linnaeus, 1766) & 8 & 0.118 & $26,27,28,29,30,31,35,50$ \\
\hline & $\begin{array}{l}\text { Ophioscion punctatissimus Meek \& Hildebrand, } \\
1925\end{array}$ & 1 & 0.015 & 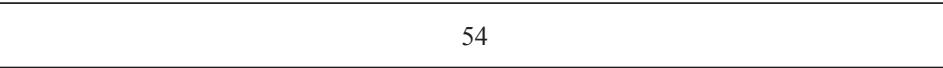 \\
\hline & Pareques acuminatus (Bloch \& Schneider, 1801) & 1 & 0.015 & 35 \\
\hline & Pogonias cromis (Linnaeus, 1766) & 48 & 0.706 & $\begin{array}{c}2,3,4,5,6,10,11,12,13,14,15,16,17,18,19,20,21,22,23,24,25,26,27,28,29, \\
30,31,32,34,35,36,37,38,42,48,49,52,53,54,55,57,58,59,60,62,63,64,68 \\
\end{array}$ \\
\hline & Nebris microps Cuvier, 1830 & 1 & 0.015 & 59 \\
\hline & Stellifer brasiliensis (Schultz, 1945) & 1 & 0.015 & 54 \\
\hline & Stellifer rastrifer (Jordan, 1889) & 2 & 0.029 & 32,54 \\
\hline & Umbrina canosai Berg, 1895 & 1 & 0.015 & 29 \\
\hline & Umbrina coroides Cuvier, 1830 & 1 & 0.015 & 42 \\
\hline
\end{tabular}


Mendes, A.B. et al.

Table 2. Continued...

\begin{tabular}{|c|c|c|c|c|}
\hline Family & Species & $\mathbf{F}$ & Relative F & Midden(s) \\
\hline \multirow{3}{*}{ Scombridae } & Katsuwonus pelamis (Linnaeus, 1758) & 2 & 0.029 & 8,47 \\
\hline & $\begin{array}{c}\text { Scomberomorus cf. brasiliensis Collette, Russo \& } \\
\text { Zavala-Camin, } 1978\end{array}$ & 2 & 0.029 & 62,63 \\
\hline & Thunnus cf. atlanticus (Lesson, 1831) & 1 & 0.015 & 64 \\
\hline \multirow{5}{*}{ Serranidae } & Cephalopholis fulva (Linnaeus, 1758) & 4 & 0.059 & $54,62,63,64$ \\
\hline & Epinephelus itajara (Lichtenstein, 1822) & 4 & 0.059 & $54,62,63,64$ \\
\hline & Epinephelus morio (Valenciennes, 1828) & 6 & 0.088 & $3,47,54,62,63,64$ \\
\hline & Hyporthodus niveatus (Valenciennes, 1828) & 2 & 0.029 & 26,29 \\
\hline & Mycteroperca acutirostris (Valenciennes, 1828) & 2 & 0.029 & 63,64 \\
\hline \multirow{5}{*}{ Sparidae } & Archosargus probatocephalus (Walbaum, 1792) & 20 & 0.294 & $2,3,4,5,6,7,9,39,53,54,55,56,57,58,59,60,62,63,64,66$ \\
\hline & Archosargus rhomboidalis (Linnaeus, 1758) & 8 & 0.118 & $3,5,26,28,29,30,31,35$ \\
\hline & Calamus pennatula Guichenot, 1868 & 2 & 0.029 & 54,62 \\
\hline & Diplodus argenteus (Valenciennes, 1830) & 2 & 0.029 & 9,60 \\
\hline & Pagrus pagrus (Linnaeus, 1758) & 8 & 0.118 & $26,28,29,30,35,47,49,53$ \\
\hline \multirow{2}{*}{ Sphyraenidae } & Sphyraena barracuda (Edwards, 1771) & 1 & 0.015 & 5 \\
\hline & Sphyraena guachancho Cuvier, 1829 & 1 & 0.015 & 3 \\
\hline \multirow{3}{*}{ Tetraodontidae } & Lagocephalus laevigatus (Linnaeus, 1766) & 24 & 0.353 & $2,3,4,5,6,7,8,9,26,28,35,39,42,49,54,55,56,58,59,60,61,62,63,67$ \\
\hline & Sphoeroides spengleri (Bloch, 1785) & 5 & 0.074 & $2,3,4,5,6$ \\
\hline & Sphoeroides testudineus (Linnaeus, 1758) & 5 & 0.074 & $2,3,4,5,6$ \\
\hline Trichiuridae & Trichiurus lepturus Linnaeus, 1758 & 17 & 0.250 & $2,3,4,5,6,8,42,47,53,54,55,58,59,60,62,63,65$ \\
\hline Triglidae & Prionotus punctatus (Bloch, 1793) & 1 & 0.015 & 1 \\
\hline
\end{tabular}

Table 3. Marine fish of class Chondrichthyes from middens in the Southeast Region of Brazil with their absolute (F) and relative (relative F) frequencies of occurrence as well as the midden(s) in which they were recorded. The codes representing the middens are defined in Table 1.

\begin{tabular}{|c|c|c|c|c|}
\hline Family & Species & $\mathbf{F}$ & Relative F & Midden(s) \\
\hline \multirow{2}{*}{ Alopiidae } & Alopias superciliosus Lowe, 1841 & 2 & 0.029 & 3,50 \\
\hline & Alopias vulpinus (Bonnaterre, 1788) & 6 & 0.088 & $3,53,55,56,58,60$ \\
\hline \multirow{17}{*}{ Carcharhinidae } & Carcharhinus acronotus (Poey, 1860) & 8 & 0.118 & $1,3,5,42,54,62,63,64$ \\
\hline & Carcharhinus altimus (Springer, 1950) & 2 & 0.029 & 3,9 \\
\hline & Carcharhinus brachyurus (Günther, 1870) & 2 & 0.029 & 3,5 \\
\hline & Carcharhinus brevipinna (Müller \& Henle, 1839) & 8 & 0.118 & $3,8,26,29,42,47,49,50$ \\
\hline & Carcharhinus falciformis (Müller \& Henle, 1839) & 1 & 0.015 & 3 \\
\hline & Carcharhinus leucas (Müller \& Henle, 1839) & 10 & 0.147 & $3,45,54,55,56,58,59,60,62,63$ \\
\hline & Carcharhinus limbatus (Müller \& Henle, 1839) & 11 & 0.162 & $1,3,4,5,6,8,45,54,62,63,64$ \\
\hline & Carcharhinus longimanus (Poey, 1861) & 2 & 0.029 & 3,45 \\
\hline & Carcharhinus obscurus (Lesueur, 1818) & 10 & 0.147 & $3,8,26,29,54,55,56,58,62$ \\
\hline & Carcharhinus perezii (Poey, 1876) & 1 & 0.015 & 3 \\
\hline & Carcharhinus plumbeus (Nardo, 1827) & 10 & 0.147 & $1,3,5,6,26,45,47,55,56,58$ \\
\hline & Carcharhinus porosus (Ranzani, 1839) & 5 & 0.074 & $1,3,4,5,6$ \\
\hline & Negaprion brevirostris (Poey, 1868) & 9 & 0.132 & $1,2,3,4,5,6,8,29,42$ \\
\hline & Prionace glauca (Linnaeus, 1758) & 9 & 0.132 & $44,54,55,56,57,58,59,60,62$ \\
\hline & Rhizoprionodon lalandii (Müller \& Henle, 1839) & 4 & 0.059 & $3,4,5,6$ \\
\hline & Rhizoprionodon porosus (Poey, 1861) & 3 & 0.044 & $3,4,5$ \\
\hline & Galeocerdo cuvier (Péron \& Lesueur, 1822) & 23 & 0.338 & $1,2,3,4,5,6,7,8,30,42,44,45,46,47,54,55,56,58,59,60,62,63,64$ \\
\hline \multirow{3}{*}{ Dasyatidae } & Bathytoshia centroura (Mitchill, 1815) & 6 & 0.088 & $3,7,26,29,39,50$ \\
\hline & Hypanus americanus (Hildebrand \& Schroeder, 1928) & 2 & 0.029 & 54,63 \\
\hline & Hypanus guttatus (Bloch \& Schneider, 1801) & 1 & 0.015 & 3 \\
\hline Ginglymostomatidae & Ginglymostoma cirratum (Bonnaterre, 1788) & 3 & 0.044 & $54,62,63$ \\
\hline Gymnuridae & Gymnura altavela (Linnaeus, 1758) & 1 & 0.015 & 7 \\
\hline \multirow{4}{*}{ Lamnidae } & Lamna nasus (Bonnaterre, 1788) & 7 & 0.103 & $1,3,4,5,6,42,47$ \\
\hline & Carcharodon carcharias (Linnaeus, 1758) & 23 & 0.338 & $1,2,3,4,5,6,7,8,26,29,42,45,47,50,54,55,56,58,59,60,62,63,64$ \\
\hline & Isurus oxyrinchus Rafinesque, 1810 & 15 & 0.221 & $3,7,8,37,39,42,45,47,54,55,56,58,60,62,63$ \\
\hline & Isurus paucus Guitart, 1966 & 1 & 0.015 & 7 \\
\hline \multirow{4}{*}{ Myliobatidae } & Aetobatus narinari (Euphrasen, 1790) & 24 & 0.353 & $\begin{array}{c}3,8,9,26,28,29,30,31,35,39,42,47,49,50,54,55,56,58,59,60, \\
61,62,63,64\end{array}$ \\
\hline & Myliobatis goodei Garman, 1885 & 5 & 0.074 & $54,55,56,58,59$ \\
\hline & Rhinoptera bonasus (Mitchill, 1815) & 10 & 0.147 & $7,39,54,55,56,58,59,60,62,63$ \\
\hline & Rhinoptera brasiliensis Müller, 1836 & 5 & 0.074 & $54,59,60,61,62$ \\
\hline Odontaspididae & Carcharias taurus Rafinesque, 1810 & 26 & 0.382 & $\begin{array}{c}1,2,3,4,5,6,7,8,35,40,41,42,43,45,47,50,53,54,55,56,57 \\
58,59,60,62,63\end{array}$ \\
\hline \multirow{4}{*}{ Sphyrnidae } & Sphyrna lewini (Griffith \& Smith, 1834) & 2 & 0.029 & 3,60 \\
\hline & Sphyrna mokarran (Rüppell, 1837) & 2 & 0.029 & 3,29 \\
\hline & Sphyrna tiburo (Linnaeus, 1758) & 2 & 0.029 & 54,59 \\
\hline & Sphyrna zygaena (Linnaeus, 1758) & 4 & 0.059 & $3,5,47,59$ \\
\hline
\end{tabular}




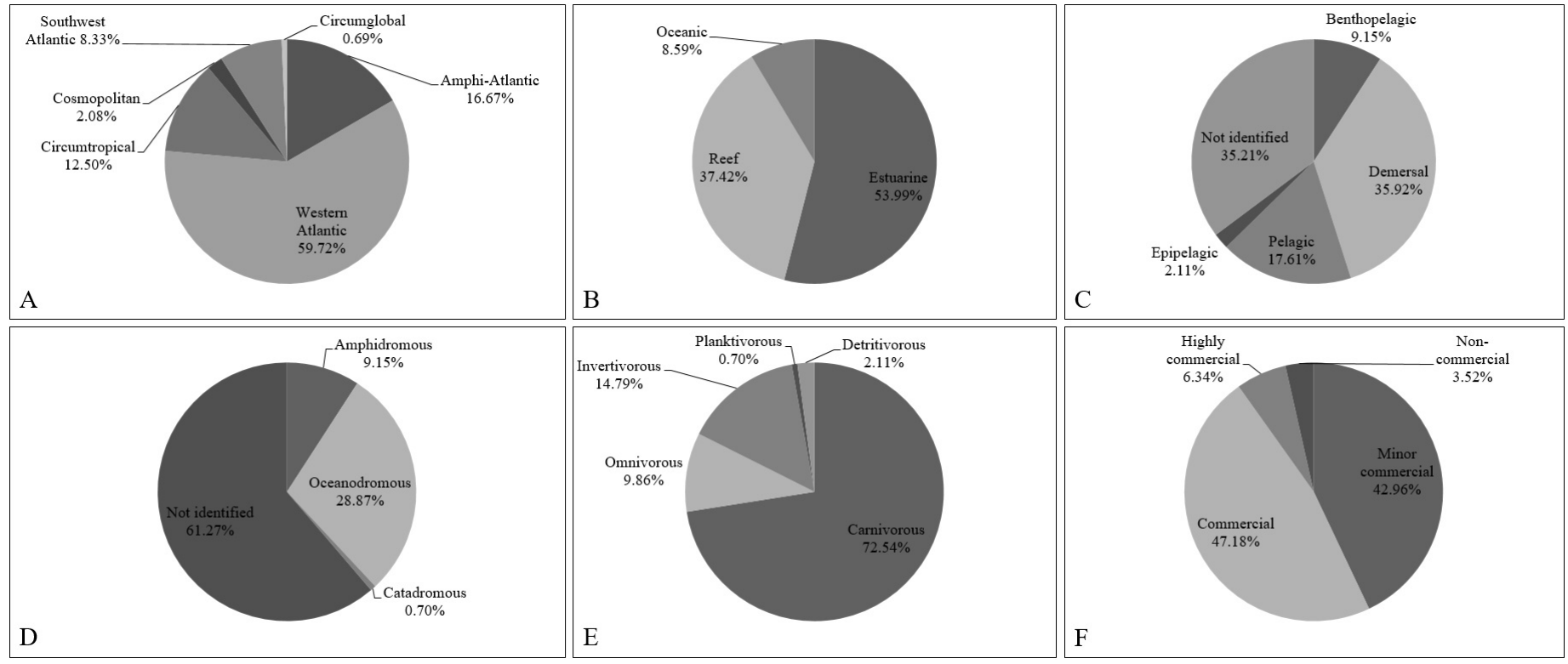

Figure 3. Biogeographical, ecological and economic data for the inventoried fish. A = Distribution; $\mathrm{B}=$ Environment; $\mathrm{C}=\mathrm{Habit} ; \mathrm{D}=\mathrm{Behaviour}$; $\mathrm{E}=\mathrm{Feeding}$ guild; $\mathrm{F}=$ Commercial value.

Table 4. Number of species from current fish inventories and middens from Rio de Janeiro (RJ), São Paulo (SP), Espírito Santo (ES) and the entire Southeast Region.

\begin{tabular}{ccccc}
\hline n spp. & RJ & SP & ES & Southeast Region \\
\hline Overall Past & 104 & 90 & 6 & 142 \\
Overall Present & 591 & 578 & 282 & 808 \\
Commercial Past & 101 & 87 & 6 & 137 \\
Commercial Present & 412 & 340 & 196 & 488 \\
Past Exclusive & 13 & 15 & 4 & 5 \\
\hline
\end{tabular}

\section{Discussion}

Middens are artificial accumulations of wildlife and cultural remains that were built by groups of pre-colonial humans during the Holocene (Lima et al. 2003, Mendes et al. 2014). Therefore, the species compositions of the organisms found in middens are presumably non-random and biased samples of natural biological communities at those sites because they were influenced or determined by various cultural factors, including food preference, the technological level of the fishing gear, harvesting and hunting artefacts, food taboos, funerary or ritualistic practices and how the materials were discarded and/or used. Therefore, some researchers believe that the faunal data from middens have selectivity biases that complicate any related inferences about ecosystems and their biodiversity (Baisre 2010, Rodrigues et al. 2016a). However, the comparison between the inventory of the marine fish identified in middens and those from general surveys of ichthyological fauna showed no significant differences in the number of species either with or without commercial value, indicating that middens contain data that are not solely applicable to prehistoric fisheries. Such wildlife remains apparently represent the fauna existing at the time that the archaeological sites were created but are also repositories of broader biodiversity data, despite any bias associated with the composition of zoo-archaeological remains (Lindbladh et al. 2007, Froyd \& Willis 2008).

In a recent study using taxonomic tests, Faria et al. (2014) showed that the malacological taxonomic diversity in the Tarioba midden (Rio de Janeiro, Brazil) was not significantly different from that of a comprehensive list of the mollusc species from the entire coast of the state of Rio de Janeiro. Those findings and the results of the present study suggest that middens may contain key indicators of past biodiversity, despite their limitations, and furthermore, some studies of middens have shown detectable changes in species composition over time (Dalzell 1998, Lotze \& Milewski 2004, Rosenberg et al. 2005, Maschner et al. 2008, Souza et al. 2016).

Studies focused on inventorying the fish species in middens have usually involved a limited number of sites (sampling areas). For example, Kloker et al. (2010) recorded 17 fish species from two sites on the south coast of Brazil (Santa Catarina state). In contrast, as part of a greater sampling effort, Lopes et al. (2016) recorded 97 fish species in 13 sites located on the coast of Rio de Janeiro, and in this study, the study area corresponded to 68 middens distributed over $1,000 \mathrm{~km}$ of the southeast coast of Brazil.

In using even greater effort than that undertaken by Lopes et al. (2016), this study used data which came from different kinds of publications (scientific articles, books, thesis, dissertations, monographs, technical reports etc) encompassing 48 years (1968-2016) of studies in the field. Therefore, the data compiled here came from a myriad of objectives and methodologies, for example, primary data which came from excavations were originated since superficial sampling till total material recovery. Following excavation the material recovered were dry sieved or under current water using different mesh sizes ( 2 to $10 \mathrm{~mm}$ ). Identification of the zoo-archaeological vestiges was done by handling different manuals and reference collections. Due to that the list recovered was carefully scrutinized. Criteria such as using only the more inclusive taxa and species with biogeographic range defined were used. Furthermore, the Linean definition of the species was fully checked for ambiguities and classification changes. In using these criteria any inconvenience related to the heterogeneity of sources were 
surpassed and the methodological choice gave an extensive baseline of fishes biodiversity during the Holocene in the Brazilian Coast.

The species richness from the midden fish inventory in this study was lower than that of current fish checklists (Bizerril \& Costa 2001, Menezes 2011, speciesLink), which likely resulted from the selected sampling methods. All current checklists were developed from extensive bibliographical surveys that included studies that employed diverse methods (fishing records, scientific collections, the testimony of scientific experts, museum collection documents and environmental monitoring) as well as data accumulated from several years of academic research. In contrast, the midden records were fundamentally related to prehistoric fisheries or related cultural activities (fishing gear, ritualistic symbols, ornaments and artefacts) as well as by-catch. In all cases, the midden fish records were always informed by an understanding of prehistoric culture; that is, the midden fish inventory was constructed with data from archaeological studies of fishing cultures and thus focused on a limited number of target species.

Regarding the target species, the occurrence frequencies of $M$. furnier $i$ and $P$. cromis in middens indicate that they were preferentially fished species. Barbosa-Guimarães (2013) observed that M. furnieri was the main fish species in Saquarema middens (Rio de Janeiro, Brazil) and thus inferred that it was the primary food of the midden peoples of that region. In turn, Souza Cunha et al. (1981) notes the presence of Pogonias sp. in the midden Sambaqui de Camboinhas (Niterói, state of Rio de Janeiro, Brazil), and the remains of that genus are commonly found in coastal middens (Kneip et al. 1975). Lopes et al. (2016) recorded M. furnieri and P. cromis as two of the three most common species in their study of Rio de Janeiro middens, and these two species are currently considered key fishery resources in the Southeast Region of Brazil (Mulato et al. 2015, Santos et al. 2016). Furthermore, estuarine, demersal and carnivorous species typically have significant commercial value, thus composing key fishing stocks (Tacon 1994, Santos \& Câmara 2002, Haimovici et al. 2014), and it can be deduced that such species were critical fishing resources for midden peoples in the past.

On the one hand, the presence of species such as M. furnieri and P. cromis, which occur at high relative frequencies in middens, indicates that midden fish inventories contain prehistoric fishery data, but the numbers of fish with and without commercial value in middens are not significantly different from those in checklists of ichthyological fauna for the same sites. Moreover, the high number of exclusive species with low frequencies of occurrence in middens corroborates the hypothesis that midden fish records contain key data on Holocene ichthyological fauna and that such species are most likely by-catch. Additionally, the occurrence frequencies show that it is unlikely that midden peoples from neighbouring regions consumed different or unique species. Thus, the results from this study indicate that middens contain data on midden fishing and culture as well as past biodiversity.

Biodiversity inventories are essential for establishing baselines that inform species management and conservation measures (Gordillo et al. 2014), especially those related to endangered species, including fish. Currently, the effects of overfishing, pollution, invasive species and other ecological impacts have reduced marine ichthyological diversity (Povey \& Keough 1991, Brosnan \& Crumrine 1994, Polunin \& Roberts 1996, Costello et al. 2010). Therefore, the study of ichthyological midden remains is a key tool for understanding prehistoric biodiversity, enabling the establishment of a historical perspective and thus more complete baselines to inform more effective management measures and reduce the threat of extinction currently faced by marine fishes.

In summary, the midden inventory of the Holocene marine fish of the southeast coast of Brazil contains data on prehistoric fisheries, which is supported by the high number of species with low frequencies of occurrence (or unique species) as well as by chi-squared tests that failed to show significant differences between the midden fish inventory and current ichthyofauna checklists. Thus, the results from this study clearly indicate that zoo-archaeological fish remains are key evidence of Holocene biodiversity.

\section{Acknowledgments}

The authors would like to thank CAPES (Coordenação de Aperfeiçoamento de Pessoal de Nível Superior) for financial support and Pos-doc Scholarship for MRD (Programa Nacional de Pós-Doutorado-PNPD) and Masters Scholarship for ABM.

\section{Author Contributions}

Augusto Barros Mendes: Contribution to data collection, contribution to data analysis and interpretation, contribution to manuscript preparation and contribution to critical revision, adding intelectual content.

Michelle Rezende Duarte: Contribution to data analysis and interpretation, contribution to manuscript preparation and contribution to critical revision, adding intelectual content

Edson Pereira da Silva: Substantial contribution in the concept and design of the study, contribution to data collection, contribution to data analysis and interpretation, contribution to manuscript preparation and contribution to critical revision, adding intelectual content

\section{Conflicts of interest}

The authors declare that there is no conflict of interest related to the publication of the data in this article.

\section{References}

AMARAL, A.Z.C. \& JABLONSKY S. 2005. Conservation of marine and coastal biodiversity in Brazil. Conserv. Biol. 19:625-631.

ARRUDA, R.S.V., SILVA, V.C.F., FIGOLS, F.A.B. \& ANDRADE, D. 2000 Os saberes tradicionais e a biodiversidade no Brasil. In: Biodiversidade e comunidades tradicionais no Brasil (A.C. Diegues, ed). Núcleo de Pesquisas Sobre Populações Humanas e Áreas Úmidas Brasileiras, Ministério do Meio Ambiente, Conselho Nacional de Pesquisa, São Paulo.

BAISRE, J. 2010. Setting a baseline for Caribbean fisheries. J. Isl. Coast. Archaeol 5:120-147.

BARBOSA-GUIMARÃES, M. 2013. Fishing strategies among prehistoric populations at Saquarema Lagoonal Complex, Rio de Janeiro, Brazil. An. Acad. Bras. Cienc. 85:415-429.

BEAUCLAIR, M., DUARTE, M.R. \& SILVA, E.P. 2016. Sambaquis (shell mounds) and mollusk diversity in the past history of Araruama Lagoon, Rio de Janeiro, Brazil. Pan-Am. J. Aquat. Sci. 11:47-59.

BITTENCOURT, J.S., KUCHENBECKER, M., VASCONCELOS, A.G. \& MEYER, K.E.B. 2015. O registro fóssil das coberturas sedimentares do Cráton do São Francisco em Minas Gerais. Geonomos 23:39-62.

BIZERRIL, C.R.F.S. \& COSTA, P.A.S. 2001. Peixes marinhos do estado do Rio de Janeiro. FEMAR-SEMADS, Rio de Janeiro.

BROSNAN, D.M. \& CRUMRINE, L.L. 1994. Effects of human trampling on marine rocky shore communities. J. Exp. Mar. Biol. Ecol. 177:79-97.

COSTA, S.A.R.F., LUZ, Z.A.S., SILVEIRA, M.I. \& MORAES-SANTOS, H.M. 2012. Contribuição à zooarqueologia do Sambaqui do Moa: novos vestígios ictiológicos. Rev. Mus. Arqueol. Etnol. 22:51-65.

COSTELlO, M.J., COLL, M., DANOVARO, R., HALPIN, P., OJAVEER, H. \& MILOSLAVICH, P. 2010. A census of marine biodiversity knowledge, resources, and future challenges. PLoS One 5(8): e12110. https://doi.org/10.1371/journal. pone.0012110 (last access on 15/06/2017)

DALZELL, P. 1998. The role of archaeological and cultural-historical records in long-range coastal fisheries resources management strategies and policies in the Pacific Island. Ocean Coast. Manage. 40:237-252. 
DEBLASIS, P.A.D., KNEIP, A., GIANINNI, P.C., GASPAR, M.D. \& SCHEELYBERT, R. 2007. Sambaquis e paisagem: dinâmica natural e arqueologia regional no litoral do sul do Brasil. Arqueologia Suramericana 3:29-61.

FARIA, R.G.S., SILVA, E.P. \& SOUZA, R.C.C.L. 2014. Biodiversity of marine molluscs from Sambaqui da Tarioba, Rio das Ostras, Rio de Janeiro (Brazil). Revista Chilena de Antropologia 29:49-54.

FIGUTI, L. 1993. O homem pré-histórico, o molusco e o sambaqui: considerações sobre a subsistência dos povos sambaquieiros. Rev. Mus. Arqueol. Etnol. 3:67-80.

FIGUTI, L. 1998. Estórias de arqueo-pescador: considerações sobre a pesca nos sítios de grupos pescadores-coletores do litoral. Revista de Arqueologia 11:57-70.

FROESE, R. \& PAULY, D. (eds) 2017. FishBase. World Wide Web electronic publication. Available from: http://fishbase.org, version 10/2017.

FROYD, C.A. \& WILLIS, K.J. 2008. Emerging issues in biodiversity \& conservation management: The need for a palaeoecological perspective. Quaternary Sci. Rev. 27:1723-1732.

FURON, R. 1969. La distribución de los seres. Editorial Labor, Barcelona.

GASPAR, M.D. 1998. Considerations about the sambaquis of the Brazilian coast. Antiquity 72:592-615.

GONZALEZ, M.M.B. 2005. Use of Pristis spp. (Elasmobranchii: Pristidae) by hunter-gatherers on the Coast of São Paulo, Brazil. Neotrop. Ichthyol 3:421-426.

GORDILLO, S., BAYER, S.B, BORETTO, B. \& CHARÓ, M. 2014. Mollusk shells as bio-geo-archives: evaluating environmental changes during the Quaternary. Springer, Berlin/Heidelberg.

HAIMOVICI, M., FILHO, J.M.A. \& SUNYE, P.S. (eds) 2014. A pesca marinha e estuarina no Brasil: estudos de caso multidisciplinares. Editora da FURG, Rio Grande.

KLOKLER, D., VILLAGRÁN, X.S., GIANNINI, P.C.F., PEIXOTO, S. \& DEBLASIS, P. 2010. Juntos na costa: zooarqueologia e geoarqueologia de sambaquis do litoral sul catarinense. Rev. Mus. Arqueol. Etnol. 20:53-75.

KNEIP, L.M., CRANCIO, F. \& FRANCISCO, B.H.R. 1988. O Sambaqui da Beirada (Saquarema, RJ): aspectos culturais e paleoambientais. Revista de Arqueologia 5:41-54.

KNEIP, L.M., CUNHA, F.L.S., COELHO, A.C.S. \& MELLO B.E.M. 1975. O "Sambaqui do Forte": correlações arqueológicas, geológicas e faunísticas (Cabo Frio, RJ-Brasil). An. Acad. Bras. Cienc. 47:91-97.

KNOWLTON, N. \& JACKSON, J.B.C. 2008. Shifting baselines, local impacts, and global change on coral reefs. PLoS Biol. 6(2):e54. https://doi: 10.1371/ journal.pbio.0060054 (last access on 15/06/2017)

LACERDA, L.D., ARAUJO, D.S.D., CERQUEIRA, R. \& TURCQ, B. (eds) 1984. Restingas: origem, estrutura, processos. CEUFF, Niterói.

LEWINSOHN, T.M. \& PRADO, P.I. 2005. Quantas espécies há no Brasil? Megadiversidade 1:36-42.

LIMA, T.A. 2000. Em busca dos frutos do mar: os pescadores-coletores do litoral centro-sul do Brasil. Rev. USP 44:270-327.

LIMA, T.A., MACARIO, K.D., ANJOS, R.M., GOMES, P.R.S., COIMBRA, R.S. \& ELAMORE, E. 2003. AMS dating of early shellmounds of the southeastern Brazilian coast. Braz. J. Phys. 33:276-279.

LINDBLADH, M., BRUNET, J., HANNON, G., NIKLASSON, M., ELIASSON, P., ERIKSSON, G. \& EKSTRAND, A. 2007. Forest history as a basis for ecosystem restoration: a multidisciplinary case study in a south Swedish temperate landscape. Restor. Ecol. 15:284-295.

LOPES, M.S., BERTUCCI, T.C.P., RAPAGNÃ, L., TUBINO, R.A., MONTEIRONETO, C., TOMAS, A.R.G., TENÓRIO, M.C., LIMA, T.A., SOUZA, R.C.C.L., CARRILLO-BRICEÑO, J.D., HAIMOVICI, M., MACARIO, K.D., CARVALHO, C. \& SOCORRO, A.O. 2016. The path towards endangered species: prehistoric fisheries in Southeastern Brazil. PLoS One 11(6):e0154476. https://doi: 10.1371/ journal.pone.0154476 (last access on 15/06/2017)

LOTZE, H.K. \& MILEWSKI, I. 2004. Two centuries of multiple human impacts and successive changes in a North Atlantic food web. Ecol. Appl. 14:1428-1447.

MASCHNER, H.D.G., BETTS, M.W., REEDY-MASCHNER, K.L. \& TRITES, A.W. 2008. A 4500-year time series of Pacific cod (Gadus macrocephals) size and abundance: archaeology, oceanic regime shifts, and sustainable fisheries. Fish. Bull. 104:386-394.
MENDES, A.B., SILVA, E.P. \& SOUZA, R.C.C.L. 2014. Biodiversity of marine fishes from Shellmounds of Ilha Grande Bay, Rio de Janeiro, Brazil. Revista Chilena de Antropologia 29:55-59.

MENEZES, N.A. 2011. Checklist dos peixes marinhos do Estado de São Paulo, Brasil. Biota Neotrop. 11:33-46.

MULATO, I. P., CORREAA, B., \& VIANNA, M. 2015. Distribuição espaço-temporal de Micropogonias furnieri (Perciformes, Sciaenidae) em um estuário tropical no Sudeste do Brasil. Bol. Inst. Pesca 41, 1-18.

PINNEGAR, J.K. \& ENGELHARD, G.H. 2008. The "shifting baseline" phenomenon: a global perspective. Rev. Fish Biol. Fisher. 18:1-16.

POLUNIN, N.V.C. \& ROBERTS, C.M. (eds) 1996. Reef fisheries. Chapman \& Hall, London.

POVEY, A. \& KEOUGH, M.J. 1991. Effects of trampling on plant and animal populations on rocky shores. Oikos 61:355-368.

PRUMMEL, W., \& HEINRICH, D. 2005. Archaeological evidence of former occurrence and changes in fishes, amphibians, birds, mammals and molluscs in the Wadden Sea area. Helgoland Mar. Res. 49:55-70.

REITZ, E.J. \& WING, E.S. 2008. Zooarchaeology. Cambridge University Press, New York.

RITTER, M.N. \& ERTHAL, F. 2016. Time-averaging e suas implicações para o registro fóssil marinho. Terrae Didatica 12:81-103.

RODRIGUES, F.B., DUARTE, M.R., SOUZA, R.C.C.L., SOARES-GOMES, A. \& SILVA, E.P. 2016a. Holocene crustaceans from the Tarioba shell mound, Rio das Ostras, Rio de Janeiro, Brazil. Check List 12:1-5.

RODRIGUES, F.B., GAROFALO, R., SOUZA, R.C.C.L., TAVARES, M.D.S. \& SILVA, E.P. 2016b. Coleção de subfósseis de sambaquis do Laboratório de Genética Marinha e Evolução-UFF. Scientia Plena 12:1-14.

ROSA, R.S. \& LIMA, F.C.T. 2008. Os peixes brasileiros ameaçados de extinção. In: Livro vermelho da fauna brasileira ameaçada de extinção: Peixes (A.B.M. Machado, G.M. Drummond \& A.P. Paglia, eds). Ministério do Meio Ambiente, Brasília.

ROSENBERG, A.A., BOLSTER, W.J., ALEXANDER, K.E., LEAVENWORTH, W.B., COOPER, A.B. \& MCKENZIE, M.G. 2005. The history of ocean resources: modeling cod biomass using historical records. Front. Ecol. Environ. 3:84-90.

SANTOS, P.R.S., EINHARDT, A.C.M.C. \& VELASCO, G. 2016. A pesca artesanal da miragaia (Pogonias cromis, Sciaenidae) no estuário da Lagoa dos Patos, Brasil. Bol. Inst. Pesca 42:89-101.

SANTOS, T.C.C. \& CÂMARA, J.B.D. (eds) 2002. GEO Brasil 2002: perspectivas do meio ambiente no Brasil. Edições IBAMA, Brasília.

SCHEEL-YBERT, R., BIANCHINI, G.F. \& DEBLASIS, P. 2009. Registro de mangue em um sambaqui de pequeno porte do litoral sul de Santa Catarina, Brasil, a cerca de 4900 anos cal BP, e considerações sobre o processo de ocupação do sítio Encantada III. Rev. Mus. Arqueol. Etnol. 19:103-118.

SILVA, E.P., SOUZA, R.C.C.L., ARRUDA, T.A. \& DUARTE, M.R. 2016. Sambaquis: mostra da biodiversidade pré-histórica. Ciência Hoje 57:30-33.

SILVEIRA, L.B., BEISIEGEL, B.M., CURCIO, F.F., VALDUJO, P.H., DIXO, M., VERDADE, V.K., MATTOX, G.M.T. \& CUNNINGHAM, P.T.M. 2010. Para que servem os inventários de fauna? Cad. Est. Av. 24:173-207.

SIQUEIRA, T., BINI, L.M., THOMAZ, S.M. \& FONTANETO, D. 2015. Biodiversity analyses: are aquatic ecologists doing any better and differently than terrestrial ecologists? Hydrobiologia 750: 5-12.

SOUZA CUNHA, F.L., VOGEL, M.A.C., VERÍSSIMO, S.G. \& MAGALHÃES, R.M.M. 1981. Restos de vertebrados do Sambaqui de Camboinhas. In: Pesquisas arqueológicas no litoral de Itaipu, Niterói, RJ (L.M. Kneip, L. Pallestrini \& F.L. Souza Cunha, eds). Cia de Desenvolvimento Territorial, Rio de Janeiro.

SOUZA, R.C C L., LIMA, T A. \& SILVA, E.P. 2010a. Holocene molluscs from Rio de Janeiro state coast, Brazil. Check List 6:301-308.

SOUZA, R.C.C.L., LIMA, T.A., DUARTE, M.R. \& SILVA, E.P. 2016. Changes in patterns of biodiversity of marine mollusks along the Brazilian coast during the late Holocene inferred from shell-mound (sambaquis) data. Holocene 26:1802-1809.

SOUZA, R.C.C.L., TRINDADE, D.C., DECCO, J., LIMA, T.A. \& SILVA, E.P. 2010b. Archaeozoology of marine mollusks from Sambaqui da Tarioba, Rio das Ostras, Rio de Janeiro, Brazil. Zoologia 27:363-371. 
STAHL, P.W. 2008. The contributions of zooarchaeology to historical ecology in the neotropics. Quatern. Int. 180:5-16.

TACON, A.G.J. 1994. Feed ingredients for carnivorous fish species: alternatives to fish meal and other fisheries resources. In: FAO Fisheries Circular, 881 FAO, Roma.

VILLAGRAN, X.S. \& GIANNINI, P.C.F. 2014. Shell mounds as environmental proxies on the southern coast of Brazil. Holocene 24:1-8.

WAGNER, G.P. \& SILVA, L.A. 2014. Prehistoric maritime domain and Brazilian shellmounds. Archaeological Discovery 2:1-5. http://dx.doi.org/10.4236/ pst.2014.21001 (last access on 15/06/2017)

WALKER, M.J.C., BERKELHAMMER, M., BJÖRCK, S., CWYNAR, L.C., FISHER, D.A., LONG, A.J., LOWE, J.J., NEWNHAM, R.M., RASMUSSEN, S.O. \& WEISS, H. 2012. Formal subdivision of the Holocene Series/Epoch: A discussion paper by a Working Group of INTIMATE (Integration of ice-core, marine and terrestrial records) and the Subcommission on Quaternary Stratigraphy (International Commission on Stratigraphy). J. Quat. Sci. 27(7):649-659.

WARWICK, R.M. \& LIGHT, J. 2002. Death assemblages of molluscs on St Martin's Flats, Isles of Scilly: a surrogate for regional biodiversity? Biodivers. Conserv. 11:99-112.

WILLIS, K.J. \& BIRKS, H.J.B. 2006. What is natural? The need for a long-term perspective in biodiversity and conservation. Science 314:1261-1265.

Received: $15 / 06 / 2017$

Revised: $16 / 12 / 2017$

Accepted: 17/12/2017

Published online: 15/01/2018 